\title{
Inflammatory Markers in Hyperemesis Gravidarum
}

\author{
(D) Özlen Emekçi Özay, (D) Ali Cenk Özay \\ Near East University Faculty of Medicine, Department of Obstetrics and Gynecology, Nicosia, Cyprus
}

\section{Abstract}

Objective: This study aims to investigate the role of inflammation in the pathophysiology of hyperemesis gravidarum (HEG).

Methods: This prospective study was conducted in the Department of Obstetrics and Gynecology at Near East University Hospital between July 2019 and March 2020. The patients that were defined as HEG should have the following symptoms: Persistent nausea and vomiting, loss of $>5 \%$ of pre-pregnancy body weight, presence of at least one positive ketonuria test in a random urine analysis, fetal heartbeat positivity at 6-13 weeks of gestation, and singleton pregnancy. Total blood count including white blood cell (WBC), neutrophil (NEU), lymphocyte (LYM), hemoglobin, hematocrit, and platelet and red cell distribution width (RDW) and platelet distribution width were analyzed. NEU-to-LYM (NLR) and platelet-to-LYM ratios (PLR) were calculated.

Results: Forty-nine patients with HEG and 121 healthy women were evaluated in the first trimester of pregnancy. The healthy pregnant women and patients with HEG had similar age, gravida, parity, gestational weeks, and body mass index. WBC, NEU, RDW, C-reactive protein (CRP), and urinary ketone levels were statistically higher in the HEG group. There were no differences in terms of NLR or PLR.

Conclusion: Measurement of inflammatory markers, such as WBC, NLR, PLR, and CRP levels, might provide valuable knowledge in HEG diagnosis. NLR and PLR are acceptable, but CRP level is a better indicator for predicting the diagnosis and severity of the disease according to our study findings.

Keywords: Hyperemesis gravidarum, inflammation, C-reactive protein, neutrophil-to-lymphocyte ratio, platelet-to-lymphocyte ratio

\section{INTRODUCTION}

Nausea and vomiting are common symptoms seen in pregnancy (1). If the symptoms are mild, nausea in the first trimester can be considered as a part of normal physiological changes. Hyperemesis gravidarum (HEG), which is a severe form of nausea and vomiting in pregnancy, can cause electrolyte imbalance, dehydration, fluid and acid-base imbalance, nutrition deficiency, ketonuria, and loss of $>5 \%$ of body weight $(1,2)$. The incidence of nausea and vomiting is $85 \%$ in pregnancy, but only $0.3 \%-2 \%$ of pregnant women will have HEG $(3,4)$. The etiopathogenesis of HEG has been evaluated in many studies, but the exact cause remains unclear. The disease is considered associated with multifactorial conditions, such as maternal endocrinological, immunological, and psychological factors. In addition, placental growing and pregestational gastrointestinal status may be related to the etiology (5). Risk factors for HEG include primigravida, multiple gestation, molar pregnancy, prior unsuccessful pregnancy, and prior HEG.

Researchers had shown that inflammation is a factor in the pathophysiology of the disease and is closely related to the severity of the symptoms. The present data are not enough to explain the role of inflammation in HEG pathogenesis, but inflammation may be associated with the onset of the HEG. In literature, the role of proinflammatory cytokines, such as interleukin-6 (IL-6), tumor necrosis factor-alpha (TNF- $\alpha$ ), and C-reactive protein (CRP), has been comprehensively evaluated in HEG pathogenesis and were associated with the disease $(6,7)$. Different hematological parameters have been used to evaluate the inflammatory status of distinct disease states recently. Mean platelet volume (MPV), platelet distribution width (PDW), 
neutrophil-to-lymphocyte ratio (NLR), red cell distribution width (RDW), and platelet-to-lymphocyte ratio (PLR) are simple markers shown to reflect inflammation and disease activity in several disorders, including ulcerative colitis, spontaneous bacterial peritonitis, malignancies, and cardiovascular diseases $(8,9)$. Oxidative stress is an instability of oxidant molecules and antioxidant defenses in humans and this imbalance may result in pathological situations that include pregnancy and complications about pregnancy. It is thought that oxidative stress, which accompanies subclinical inflammation, may trigger the onset of HEG (9).

This study aims to investigate the diagnostic value of MPV, PDW, NLR, RDW, PLR, and CRP in patients with HEG.

\section{METHODS}

This prospective study was conducted in the Department of Obstetrics and Gynecology at Near East University Hospital, Faculty of Medicine, Nicosia, Cyprus, between July 2019 and March 2020 (date: 25.07.2019, 2019/71, 877). The study protocol was approved by our institutional review board, and an informed consent was obtained from all patients. A total of 49 patients with HEG and 121 healthy gestational age-matched control subjects were studied. The patients that were defined as HEG should have the following symptoms: Persistent nausea and vomiting, loss of $>5 \%$ of pre-pregnancy body weight, presence of at least one positive ketonuria test in a random urine analysis, fetal heartbeat positivity at 6-13 weeks of gestation, and singleton pregnancy. Patients with HEG were not classified into subgroups because the group consisted of patients with mild HEG. Patients who had multiple gestations, cigarette smoking, chronic diseases, thyroid disorders, gastrointestinal disorders, or urinary infections were excluded. Age, body mass index, gestational age, gravidity, and parity of each patient were recorded. The gestational age was determined by using the date of the last menstrual period, and it was confirmed by ultrasonography. Body mass index was calculated by dividing body weight in kilograms by the square of height in meters.

Total blood count including white blood cell (WBC), neutrophil (NEU), lymphocyte (LYM), hemoglobin, hematocrit, and platelet and RDW and PDW were analyzed. NLR and PLR were calculated. Using differential count, NLR was calculated by dividing the absolute NEU count by the absolute LYM count. PLR was calculated by dividing platelet count by the LYM count. Urine ketone levels, CRP, and thyroid-stimulating hormone levels were also recorded. The ketonuria was graded as $1+, 2+$, and $3+$.

\section{Statistical Analysis}

Statistical Program for Social Sciences (SPSS) version 16 (SPSS, Chicago, IL) was used for data analysis. Homogeneity of continuous variables was assessed using Shapiro-Wilk test. HEG and control patients' values were compared using independent t-test. Data were expressed as mean \pm standard deviation. Gestational age, gravida, and parity were analyzed using Mann-Whitney $U$ test, and the results are given as median (minimum-maximum). Multivariate analysis was performed for PLR, RDW, and CRP, WBC, and NEU counts. Receiver operating characteristic (ROC) curves are drawn for CRP and NEU count. $\mathrm{P}<0.05$ was considered to be statistically significant.

\section{RESULTS}

The results of 170 patients were evaluated. Forty-nine patients had HEG, and 121 were healthy women in the first trimester of pregnancy. Table 1 shows a comparison of demographic characteristics between patients with HEG and healthy controls. The healthy pregnant women and patients with HEG had a similar age, gravida, parity, gestational weeks, and body mass index. Table 2 shows the univariate analysis of the comparison of laboratory findings between patients with HEG and healthy controls. WBC, NEU, and CRP levels and RDW were statistically higher in the HEG group according to the univariate analysis (Table 2). Urinary ketone levels were evaluated by the chi-square test, and it was statistically significantly high in the HEG group compared with the control group ( $p=0.014)$. There were no differences in terms of NLR or PLR between HEG and healthy pregnant women. When we performed a multivariate analysis only, CRP levels were statistically high for patients with HEG $(p<0.001)$. Since the multivariate analysis was significant for CRP levels only, we drew the ROC curve for CRP as shown in Figure 1. Table 3 illustrates the sensitivity and specificity for CRP in the diagnosis of HEG.

Table 1. Comparison of demographic findings

\begin{tabular}{|l|l|l|l|}
\hline & $\begin{array}{l}\text { Healthy control subjects } \\
(\mathbf{n = 1 2 1})\end{array}$ & $\begin{array}{l}\text { HEG } \\
(\mathbf{n}=\mathbf{4 9})\end{array}$ & ${ }^{* \mathbf{p}}$ \\
\hline aAge (years) & $29.22 \pm 6.00$ & $28.10 \pm 4.87$ & 0.247 \\
\hline bGravida & $1(1-6)$ & $1(1-3)$ & 0.894 \\
\hline bParity & $0(0-3)$ & $0(0-2)$ & 0.931 \\
\hline aGestational weeks & $7(6-13)$ & $7(6-13)$ & 0.457 \\
\hline aBMI (kg/m²) & $23.18 \pm 1.28$ & $22.54 \pm 1.98$ & 0.319 \\
\hline $\begin{array}{l}\text { The results are given as median (minimum-maximum). *p<0.05 is significant, } \\
\text { alndependent t-test, bMann-Whitney U test. BMI: Body mass index, HEG: } \\
\text { Hyperemesis gravidarum }\end{array}$ \\
\hline
\end{tabular}




\section{DISCUSSION}

Inflammation and oxidative stress play an important role in the pathophysiology of HEG $(7,8)$. NLR, PLR, and CRP are markers that reflect acute and chronic inflammation. Therefore, the study aimed was to compare and evaluate the inflammatory markers in pregnant women with HEG and healthy pregnant women. Our findings revealed that WBC, NEU, and serum CRP levels were statistically significantly high in patients with HEG compared with healthy controls. There was no difference in terms of NLR or PLR. Beyazit et al. (10) assessed 54 patients with HEG and 58 healthy controls. Their study results showed an increase in NLR and PLR in patients with HEG. Beyazit et al. (10) explained that NLR and PLR were elevated due to a physiological immune response of circulating leukocytes to HEG-associated physical stress, which results in amplification of NEU and decrease in LYM counts. Kan et al. (11) evaluated 113 patients with HEG and 41 healthy controls. In Kan et al.'s (11) study, markedly higher inflammatory markers, NLR, PLR, and CRP levels were found in patients with HEG compared with individuals who did not experience nausea

\begin{tabular}{|c|c|c|c|}
\hline \multicolumn{4}{|c|}{ Table 2. Laboratory findings } \\
\hline & $\begin{array}{l}\text { Healthy control } \\
\text { subjects } \\
(n=121)\end{array}$ & $\begin{array}{l}\text { HEG } \\
(n=49)\end{array}$ & $* p$ \\
\hline $\mathrm{Hb}(\mathrm{g} / \mathrm{dL})$ & $12.22 \pm 1.18$ & $12.36 \pm 1.23$ & 0.487 \\
\hline Htc (\%) & $38.29 \pm 3.42$ & $38.52 \pm 3.53$ & 0.698 \\
\hline $\mathrm{WBC}\left(\mathrm{mm}^{3} / 10^{3}\right)$ & $7.98 \pm 2.05$ & $8.73 \pm 1.86$ & 0.027 \\
\hline $\operatorname{NEU}\left(\mathrm{mm}^{3} / 10^{3}\right)$ & $5.32 \pm 1.55$ & $5.93 \pm 1.52$ & 0.019 \\
\hline $\operatorname{LYM}\left(\mathrm{mm}^{3} / 10^{3}\right)$ & $2.04 \pm 0.53$ & $2.15 \pm 0.48$ & 0.208 \\
\hline $\mathrm{PLT}\left(\mathrm{mm}^{3} / 10^{3}\right)$ & $236.00 \pm 53.22$ & $251.00 \pm 56.05$ & 0.096 \\
\hline NLR & $2.77 \pm 1.03$ & $2.81 \pm 0.69$ & 0.767 \\
\hline PLR & $122.78 \pm 41.00$ & $121.75 \pm 36.37$ & 0.879 \\
\hline PDW (\%) & $19.11 \pm 1.25$ & $19.41 \pm 1.11$ & 0.150 \\
\hline RDW (\%) & $12.20 \pm 1.11$ & $12.64 \pm 1.06$ & 0.018 \\
\hline MPV (fL) & $8.57 \pm 1.42$ & $8.39 \pm 0.98$ & 0.348 \\
\hline $\mathrm{CRP}(\mathrm{mg} / \mathrm{L})$ & $0.51 \pm 0.42$ & $2.00 \pm 0.84$ & $<0.001$ \\
\hline TSH (mIU/L) & $1.45 \pm 0.97$ & $1.36 \pm 0.75$ & 0.578 \\
\hline \multicolumn{4}{|c|}{$\begin{array}{l}\text { Independent t-test. * }{ }^{*}<0.05 \text { is significant, Hb: Hemoglobin, Htc: Hematocrit } \\
\text { WBC: White blood cells, NEU: Neutrophils, LYM: Lymphocyte, PLT: Platelet count } \\
\text { NLR: Neutrophil-to-lymphocyte ratio, MPV: Mean platelet volume, PLR: Platelet } \\
\text { to-lymphocyte ratio, PDW: Platelet distribution width, RDW: Red cell distribution } \\
\text { width, CRP: C-reactive protein, TSH: Thyroid-stimulating hormone, HEG } \\
\text { Hyperemesis gravidarum }\end{array}$} \\
\hline
\end{tabular}

or vomiting. This elevation of NEU and platelet levels in complete blood counts may be explained by the augmented inflammation process in HEG pathophysiology. In addition, Kan et al. (11) divided the patients into mild, moderate, and severe according to the symptoms. The severe group had the highest levels of CRP and NLR and PLR, but only CRP showed a statistically significant positive correlation with the severity of the disease (11). Our study findings of elevated NEU and serum CRP levels promote the base of inflammation process in the pathophysiology of HEG. However, we could not show the raised levels of NLR and PLR in our results. This may be due to the new onset of HEG symptoms and severity of nausea and vomiting because our study groups were mostly formed from patients with mild or moderate HEG symptoms.

Platelets are dynamic blood particles whose primary function, along with the coagulation factors, is hemostasis or prevention of bleeding. Platelets interact with each other, as well as with leukocyte and endothelial cells, searching for sites of injury, where they become activated (12). In pregnancy, platelets

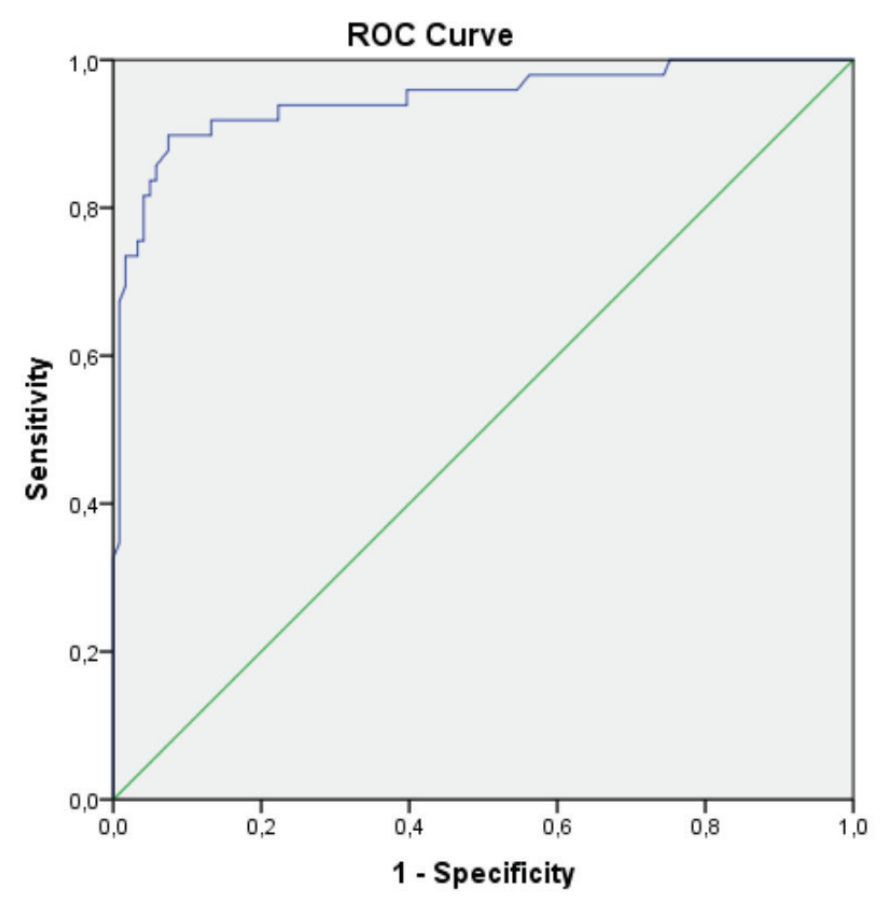

Diagonal segments are produced by ties.

Figure 1. ROC curve for CRP

ROC: Receiver operating characteristic, CRP: C-reactive protein

\begin{tabular}{|l|l|l|l|l|l|l|l|}
\hline \multicolumn{2}{|l|}{ Table 3. ROC data results for CRP and neutrophil count } \\
\hline Parameter & AUC & $\mathbf{9 5 \%}$ Cl & SE & Cut-off v value & Sensitivity & Specificity & * $\mathbf{p}$ \\
\hline CRP & 0.946 & $0.90-0.99$ & 0.021 & $1(\mathrm{mg} / \mathrm{L})$ & $90 \%$ & $91 \%$ & $<0.001$ \\
\hline *Statistically significant $(\mathrm{p}<0.05)$, ROC: Receiver operating characteristic, CRP: C-reactive protein, AUC: Area under the curve, SE: Standard error, Cl: Confidence interval \\
\hline
\end{tabular}


may be affected by many factors other than the inflammatory processes, such as hemodilution and aggregation, and these factors are generally reflected in laboratory data as a decrease. In addition, hormones, such as estrogen, whose levels increase during pregnancy, can affect platelet functions and contribute to hypercoagulability. Evidence demonstrates that platelets contribute to the inflammatory process, microbial host defense, wound healing, angiogenesis, and remodeling (13). Platelet activation can be related to platelet size. MPV and PDW reflect platelet size and activation. They are from the most widely used surrogate markers of platelet function and have been shown to reflect inflammation $(14,15)$. In literature, there is only one study to observe the relationship between MPV and PDW and existence of HEG (10). Beyazit et al. (10) found no significant difference in MPV and PDW in patients with HEG compared with healthy controls, which is in accordance with our study findings. In our study, MPV and PDW showed no statistically significant difference in patients with HEG and healthy controls, which puts forward that there should be pathological pathways related to HEG other than platelet activation.

\section{Study Limitations}

The limitations of this study are as follows. The first major limitation is that, in our study, the patients had mild-to-moderate HEG symptoms. Second, it would be worthwhile to study the serum levels of well-demonstrated proinflammatory markers, such as ILs and TNF- $\alpha$, as this would give more understanding to the pathophysiology of HEG, if oxidative markers would be assessed and compared in patients with HEG. Alternatively, one of the major strengths of our study is the availability of complete data for each patient and exclusion of patients with risk factors for HEG, such as the presence of chronic and systemic endocrine, immunologic, or gastrointestinal disease.

\section{CONCLUSION}

Measurement of inflammatory markers, such as WBC and CRP levels and NLR and PLR might provide valuable knowledge in HEG diagnosis because inflammation contributes to the pathophysiology of HEG. NLR and PLR are acceptable, but CRP level is a better indicator for predicting the diagnosis and severity of the disease according to our study findings.

\section{Ethics}

Ethics Committee Approval: This prospective study was conducted in the Department of Obstetrics and Gynecology at Near East University Hospital, Faculty of Medicine, Nicosia,
Cyprus, between July 2019 and March 2020 (date: 25.07.2019, 2019/71, 877).

Informed Consent: Was obtained from all patients.

Peer-review: Externally and internally peer-reviewed.

\section{Authorship Contributions}

Surgical and Medical Practices: A.C.Ö., Concept: A.C.Ö., Design: A.C.Ö., Data Collection or Processing: Ö.E.Ö., Analysis or Interpretation: A.C.Ö., Ö.E.Ö., Literature Search: Ö.E.Ö., Writing: Ö.E.Ö., A.C.Ö.

Conflict of Interest: No conflict of interest was declared by the authors.

Financial Disclosure: The authors declared that this study received no financial support.

\section{REFERENCES}

1. Lacasse A, Rey E, Ferreira E, Morin C, Bérard A. Nausea and vomiting of pregnancy: what about quality of life? BJOG 2008;115:1484-93.

2. Godsey RK, Newman RB. Hyperemesis gravidarum. A comparison of single and multiple admissions. J Reprod Med 1991;36:287-90.

3. Attard CL, Kohli MA, Coleman S, Bradley C, Hux M, Atanackovic G, et al. The burden of illness of severe nausea and vomiting of pregnancy in the United States. Am J Obstet Gynecol 2002;186:220-7.

4. Cunningham FG, Gant NF, Leveno KJ, Gilstrap LC, Hauth JC, Wenstrom KD, editors. Gastrointestinal Disorders. Williams Obstetrics. New York, USA: Mc Graw Hill; 2018.p.1042-57.

5. Goodwin TM. Hyperemesis gravidarum. Obstet Gynecol Clin North Am 2008;35:401-17.

6. Ismail SK, Kenny L. Review on hyperemesis gravidarum. Best Pract Res Clin Gastroenterol 2007;21:755-69.

7. Yoneyama Y, Suzuki S, Sawa R, Yoneyama K, Doi D, Otsubo Y, et al. The T-helper 1/T-helper 2 balance in peripheral blood of women with hyperemesis gravidarum. Am J Obstet Gynecol 2002;187:1631-5.

8. Verit FF, Erel 0 , Celik H. Paraoxonase-1 activity in patients with hyperemesis gravidarum. Redox Rep 2008;13:134-8.

9. Lee NM, Saha S. Nausea and vomiting of pregnancy. Gastroenterol ClinNorth Am 2011;40:309-34.

10. Beyazit F, Öztürk FH, Pek E, Ünsal MA. Evaluation of the hematologic system as a marker of subclinical inflammation in hyperemesis gravidarum: a case control study. Ginekol Pol 2017;88:315-9.

11. Kan E, Emekter E, Corbacioglu K, Safak T, Sariaydin T, Cevik Y. Evaluation of relationship between inflammatory markers and hyperemesis gravidarum in patients admitted to emergency department. Am J Emerg Med 2020;38:292-5.

12. Lopez E, Bermejo N, Berna-Erro A, Alonso N, Salido GM, Redondo PC, et al. Relationship between calcium mobilization and platelet $\alpha$ - and $\delta$-granule secretion. A role for TRPC6 in thrombin-evoked $\delta$-granule exocytosis. Arch Biochem Biophys 2015;585:75-81.

13. Golebiewska EM, Poole AW. Platelet secretion: From haemostasis to wound healing and beyond. Blood Rev 2015;29:153-62. 
14. Huczek Z, Filipiak KJ, Kochman J, Michalak M, Roik M, Piatkowsk $\mathrm{R}$, et al. Baseline platelet size is increasedin patients with acute coronary syndromes developing early stent thrombosis and predicts future residual platelet reactivity. A case-control study.Thromb Res 2010;125:406-12.
15. Ulucan Ş, Keser A, Kaya Z, Katlandur H, Özdil H, Bilgi M, et al. Association between PDW and Long Term Major Adverse Cardiac Events in Patients with Acute Coronary Syndrome. Heart Lung Circ 2016;25:29-34. 